\title{
The Impact of Sequencing and Prior Knowledge on Learning Mathematics Through Spreadsheet Applications
}

\author{
Tracey Clarke \\ Paul Ayres \\ John Sweller
}

According to cognitive load theory, instruction needs to be designed in a manner that facilitates the acquisition of knowledge in long-term memory while reducing unnecessary demands on working memory. When technology is used to deliver instruction, the sequence in which students learn to use the technology and learn the relevant subject matter may have cognitive load implications, and should interact with their prior knowledge levels. An experiment, using spreadsheets to assist student learning of mathematics, indicated that for students with little knowledge of spreadsheets, sequential instruction on spreadsheets followed by mathematics instruction was superior to a concurrent presentation. The reverse was found for students with more knowledge of spreadsheets. These results are explained in terms of cognitive load theory.
Using new technologies to enhance the learning and teaching of mathematics is highly recommended by many professional teaching associations. However, when learning from computer-based instructional material, a number of cognitive load theory (CLT) principles need to be followed to ensure that learning is maximized. While much is known about how computer-based materials should be presented to avoid negative effects such as split-attention and redundancy (e.g., see Sweller, van Merriënboer, \& Paas, 1998), less is known about the interactions associated with learning how to use technology while simultaneously learning mathematical concepts. How should instructional materials be structured so learners can employ technology in order to enhance understanding of mathematics? In this article we investigate how sequencing the learning of spreadsheet skills affects learning mathematics.

\section{CLT}

For the last two decades, CLT has been successfully employed to guide instructional design. A basic assumption of CLT is that interactions between working memory and long-term memory play a significant role in learning. When dealing with novel information, working memory is extremely limited. Humans are only able to store and process a few novel combinations of elements or chunks at any given time (Miller, 1956). In isolation, such a restriction might suggest that humans are incapable of processing complex materials. Long-term memory, by 
effectively altering the capacity and duration limits of working memory, permits complex processing. In contrast to working memory, humans can store vast quantities of information in long-term memory. In particular, it is argued by cognitive load theorists (see Paas, Renkl, \& Sweller, 2003; Sweller, 2003; Sweller et al., 1998) that information stored in long-term memory can vastly increase the capacity of working memory. The limitations of working memory only apply to novel combinations of elements. Working memory has no known limitations when dealing with previously learned information stored in long-term memory (Ericsson \& Kintsch, 1995).

Information is stored in long-term memory in the form of schemas. A schema (see Chi, Glaser, \& Rees, 1982; Larkin, McDermott, Simon, \& Simon, 1980) can be defined as a cognitive construct that allows multiple elements of information to be treated as a single element categorized according to its use. When such schemas are brought into working memory they can be treated as a single element rather than many, freeing up working memory resources, and reducing overall cognitive load. Automation (Kotovsky, Hayes, \& Simon, 1985) has a similar function: It also reduces cognitive load by reducing the burden on working memory when information can be processed automatically without conscious processing. The possession and automation of schemas enable humans to engage in complex activities in spite of a very restricted working memory. CLT proponents assume that students find many tasks difficult to learn because of the limitations of human working memory when dealing with novel material, and that problem-solving skill develops by the construction of domain-specific schemas in longterm memory (see Kalyuga, Ayres, Chandler \& Sweller, 2003; van Merriënboer \& Ayres, this issue).

Cognitive load theorists argue that overloading working memory inhibits learning, and consequently, instructional procedures are most effective when unnecessary cognitive load is kept to a minimum (for a more detailed discussion see Sweller, 2003; Sweller et al., 1998). CLT researchers have identified three sources of cognitive load during instruction: Intrinsic, extrane- ous and germane cognitive load (see Paas, Renkl et al., 2003; Sweller et al., 1998). Intrinsic cognitive load is load placed on working memory by the intrinsic nature of the materials to be learnt. Extraneous cognitive load is the load placed on working memory by the instructional design itself and germane cognitive load is the load evoked by the instructional materials that assist the process of schema formation. Whereas germane cognitive load is considered positive because working memory resources are directly focused on learning, the other two forms of cognitive load can seriously impede learning.

CLT researchers have identified strategies to reduce intrinsic cognitive load, such as isolating integrated elements in instructional materials (Pollock, Chandler, \& Sweller, 2002) and simpleto-complex sequencing of learning tasks (van Merriënboer, Kirschner, \& Kester, 2003). However, much of the research has focused on devising design strategies to reduce extraneous cognitive load (for a summary see Sweller et al., 1998; Sweller, 1999). The use of worked examples and physically integrating disparate sources of information are two such successful strategies particularly relevant to this study, and are, therefore, discussed in more detail here.

\section{Worked Examples}

Early research into CLT found that instructional formats that required problem-solving search strategies imposed a heavy working memory load that retarded learning (Sweller, 1988). To avoid such problem-solving approaches and enhance learning, instructional designers have successfully employed worked examples in a number of domains, including mathematics (see Cooper \& Sweller, 1987; Paas \& van Merriënboer, 1994; Sweller \& Cooper, 1985). A workedexample approach requires learners to study solutions to problems rather than just solve them, although in most applications of the workedexamples approach, learners also solve some problems, either in a paired format (study one, solve a similar one, see Cooper \& Sweller, 1987), or as completion problems (completion of a partial solution, see Paas \& van Merriënboer, 1994). 
Integrating Instruction

Integrating instruction was a strategy devised to avoid the split-attention effect. The split-attention effect typically occurs when instructional materials provide two sources of material, such as a diagram and some explanatory text. If both sources are needed to understand the material, the learner is forced to mentally integrate the two sources. This process increases cognitive load and is extraneous to schema acquisition and automation. Researchers have overcome this impediment to learning by devising strategies that physically integrate the two sources, thus eliminating split attention (see Tarmizi \& Sweller, 1988). From an e-learning perspective, research has shown that the split-source of a computer screen and manual can be eliminated by integrating all material into a computer manual only (Chandler \& Sweller, 1996), or onto a computer screen only (Cerpa, Chandler, \& Sweller, 1996; Moreno \& Valdez, this issue).

\section{COGNITIVE LOAD IMPLICATIONS FOR SEQUENCING THE LEARNING OF SPREADSHEET AND MATHEMATICS SKILLS}

According to CLT, to use spreadsheets effectively to learn mathematics, some principles need to be followed in the design of the instructional materials. If specific spreadsheet skills need to be learned first in order to be useful in learning mathematics, then sequencing order is critical. Both spreadsheet and mathematical tasks are high in element interactivity and intrinsic cognitive load. If both learning tasks are presented simultaneously, it is likely that cognitive load will be compounded. As a consequence, learning may be inhibited on both tasks; therefore, to maximize learning in the mathematical domain, spreadsheet skills should be mastered and consolidated first.

Recent work on presentation sequencing provides support for this argument. Kester, Kirschner, and van Merriënboer (2004; in press) and Kester, Kirschner, van Merriënboer and Baumer (2001) found that the simultaneous presentation of all necessary information, either before or during practice, had no beneficial effect on learning. However, learning was enhanced if supportive information was presented sequentially before or during practice. Furthermore, van Merriënboer et al. (2003) have argued that sequential order is particularly important in areas of high element interactivity. When using spreadsheets to learn mathematics, if students are able to learn the "necessary" (p. 9) spreadsheet skills first, schemas will be acquired in long-term memory which can be activated during application of those skills to learning new mathematical concepts. Activation of such schemas will reduce cognitive load when learning the new information. Tasks in mathematics and spreadsheets are both high in terms of element interactivity, so this argument is consistent with the conditions met in this study.

However, recent research has found that the effectiveness of instructional materials is dependent on the expertise of the learner. There is an interaction between prior knowledge levels of learners and the amount of information included in instruction. In some circumstances, information that is essential for a novice has been found to be redundant for those with more expertise. This interaction is called the expertise reversal effect because, with increasing levels of expertise, strategies that are effective for novices have been shown to be ineffective for more knowledgable students (Kalyuga et al., 2003). As a rule, inexperienced learners need much more guidance than more experienced learners in any particular domain (Mayer, 2001; Renkl \& Atkinson, 2003). Worked examples, for instance, are very effective for novices (van Merriënboer and Ayres, this issue), but as expertise develops (resulting in more sophisticated schemas), a problem-solving approach may be superior (Kalyuga, Chandler, \& Sweller, 2001).

Because of the influence of expertise, sequencing the acquisition of spreadsheet skills prior to mathematical acquisition may not be necessary. Students experienced in using spreadsheets may possess schemas that will prevent cognitive overload when simultaneously combining technology and mathematical tasks. In such a case, students may benefit from having materials structured in a concurrent format because of a reduction in temporal split attention (Mayer \& Anderson, 1991; 1992). A concur- 
rent format and its attendant reduction in split attention and cognitive load may enable students to focus on the relations between spreadsheet knowledge and mathematics.

This study, based on CLT, investigated the timing of learning spreadsheet skills in applying spreadsheet applications to mathematics. We hypothesized that students with a low-level knowledge of spreadsheets would learn mathematics more effectively if the relevant spreadsheet skills were learned prior to attempting the mathematical tasks. We also hypothesized that students experienced in spreadsheets would not need such a sequenced approach, being able to benefit from a more integrated approach, where new spreadsheet skills and mathematical concepts were learned together. To test these hypotheses, students were assigned to one of two instructional formats. The sequential group was given instructions on spreadsheets prior to applying this knowledge to learning mathematics. The concurrent group was given the spreadsheet skills and mathematical concepts in an integrated format. To test the effects of expertise, students with differing levels of experience with spreadsheets were assigned to each instructional group.

The primary goal of the study was to investigate how the given conditions would affect learning mathematics. However, a secondary goal was to investigate how these conditions would also affect learning spreadsheet skills. Consequently, both mathematics and spreadsheet skills were tested and included as dependent variables. Furthermore, a subjective measure of how difficult participants found the instruction was collected and included in the analysis.

\section{METHOD}

\section{Participants}

Ninth-grade high school students $(N=24)$ from an independent boy's school in the Sydney metropolitan area (Australia) participated in the study. Some of the students had no experience, whereas others had used spreadsheets to draw graphs, collect data, and complete simple budgets. Based on class tests, students had been assessed by the school as above average in general mathematics ability, and were following a mathematics curriculum specifically designed for students in the top $25 \%$ of the state of New South Wales. Furthermore, these students had been assessed by their school as being similar in mathematical ability, and had been grouped in the same mathematics class.

Each participant was allocated to one cell of a $2 \times 2$ between-subjects factorial design, with instructional format (sequential or concurrent treatment) as the first factor, and perceived spreadsheet ability as the second factor. To control for possible mathematical effects, students were matched in pairs on mathematical ability, and assigned to one of the treatment groups at random (both $n=12$ ). Matching was achieved by using school-based rankings. It should be noted that this matching procedure was considered an extra precaution, because the whole class were approximately of the same mathematical standard. To ascertain spreadsheet ability, students were asked to rate their current spreadsheet ability according to a 4-point scale of advanced, intermediate, beginner, and no experience. These ratings were used to classify students as high spreadsheet experience (advanced or intermediate selections; $n=14$ ) or low spreadsheet experience (beginner or no experience; $n=10$ ).

\section{Materials}

Materials were designed for instruction (acquisition phase) and testing (test phase). The mathematical purpose of the experiment was to use spreadsheets to assist the development of understanding of graphical representations of linear functions when they are given in both table and equation form. Prior to commencing the study, students were halfway through an algebraic module on coordinate geometry. Students had used formulas for finding the gradients, midpoints and distances to given sets of coordinate points, but had not applied this knowledge to graphical representations. The mathematical concepts covered in the experiment required the students to understand the relationships between the calculated values for gradient $(m)$ and $y$ intercepts $(C)$ in the $y$-inter- 
cept form $(y=m x+C)$ of the linear function, and the visual representation of such information in graphical form. Consequently, at the conclusion of the instructional period, the students should have been able to graph various forms of the linear equation, and reverse the process by calculating the linear formula from graphical representations. To create a graph (chart) of an equation in $\mathrm{Excel}^{\mathrm{TM}}$, the user must first create a table of values to be graphed. This process reinforces the fact that an equation is simply a relationship that connects independent and dependent variables, and that all three forms(a) equations, (b) tables of values, and (c) graphs_of a linear model are alternate representations of each other.

Acquisition phase. Instruction booklets were specifically designed to teach the required spreadsheet skills (entering formulas, using the function facility, and creating line graphs) and mathematical concepts. Cognitive load principles were used to integrate text and screen captures (see Figure 1) to create an effective learning environment and reduce split attention. Total self-containment of instruction was achieved by the extensive use of screen captures. Each book- let was designed to cater for three 50-min lessons.

The two instructional format groups received different booklets. For the sequential group, the instruction booklet was divided into two sections. The first section contained instructions to develop all of the necessary spreadsheet skills (similar to a how-to computer manual). The second section consisted of the mathematical content to be learned, and employed the use of the spreadsheet skills learned in the first section. Hence the mathematical concepts were learned after the development of the spreadsheet skills. The instruction booklet given to the concurrent group combined spreadsheet skill development and mathematics instructions, developing the spreadsheet skills and their application to mathematics concurrently. Table 1 summarizes the particular mathematical and spreadsheet concepts learned and the order in which they were presented over the three lessons. The order in which spreadsheet skills and mathematical concepts were introduced was identical for both groups with one exception. For the concurrent group, the spreadsheet skill "gradient and slope functions" was introduced at a later point to coincide with its mathematical applications.

Figure $1 \square$ An example of the integration of instructional texts with screen captures.

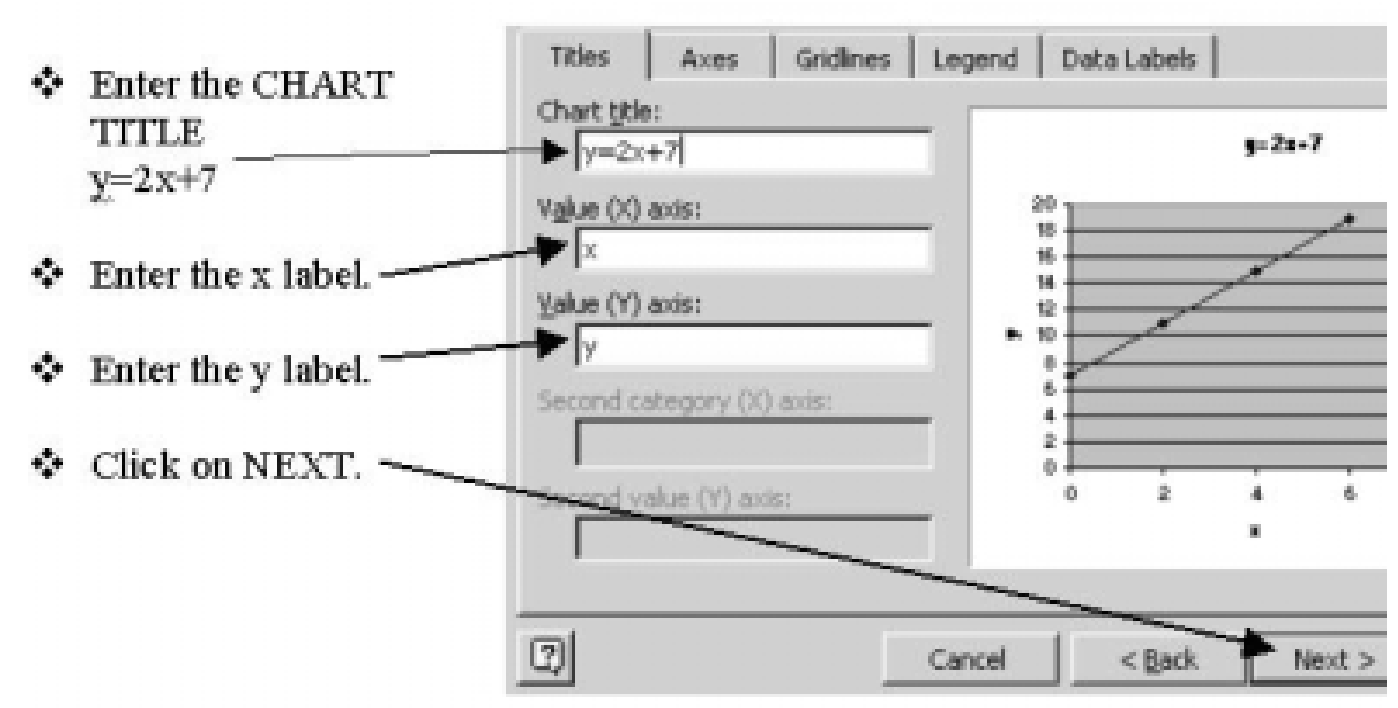


The general presentation format of both booklets was identical. Instructions requiring the participant to perform a task on the computer were clearly identified with a special bullet. Mathematical knowledge was conveyed in easily identifiable areas called MATHS SPEAK boxes. General spreadsheet information was not specifically identified. Both booklets contained the same instructions and activities. Again, to maximize learning, principles of CLT were used in the form of worked examples. Students were required to complete four tasks for each lesson, using an Excel spreadsheet by following written instructions. The written instructions were supplemented by screen captures to facilitate the ease of application (see Figure 1). Each task followed a worked example. Each successive lesson offered practice of previously learned skills, followed by additional spreadsheet skills and/or mathematical concepts.

Test phase. The test phase consisted of two sections, to assess both the mathematical concepts and spreadsheet skills learned. The mathematics section had nine problems, which could be completed by pen, paper, and calculator. The nine problems were divided into 14 parts consisting of 1 recall question, 9 application problems, and 4 synthesis problems. Each part of the mathe- matics test was assigned a value of 1 , giving a maximum score of 14 . The spreadsheet section had five problems and was completed with the use of the computer. These five problems had 10 parts, consisting of 2 recall questions, 5 application problems, and 3 synthesis problems. Each part was assigned a value of 1 , giving a maximum score of 10 . The classification of problems was determined using definitions from Bloom's Taxonomy (1956); the following examples are indicative of each category. A recall problem required students to convert a linear equation (e.g., $y=3 x+13$ ) into a spreadsheet formula. An application problem required students to use their knowledge about special functions (e.g., slope and intercept) to draw a graph. A synthesis question asked students to graph two lines (e.g., $y=x+4$ and $y=x-10$ ) and compare them. Students were then asked to explain how this comparison could have been made without drawing the lines.

Subjective measures of cognitive load. Subjective measures have been used extensively by cognitive load researchers to obtain a measure of cognitive load (see Paas \& van Merriënboer, 1993; Paas, Tuovinen, Tabbers, \& van Gerven, 2003), and were employed in this study. Students were required to rate "how easy or difficult they find

Table $1 \square$ Instructional order for each group per lesson.

\begin{tabular}{ccc}
\hline \multicolumn{2}{c}{ Concurrent Instructions } & \multicolumn{2}{c}{ Sequential Instructions } \\
Spreadsheet skills & Mathematics Skills & Spreadsheet skills $\quad$ Mathematics Skills \\
\hline
\end{tabular}

Lesson 1

1. Editing data

2. Entering formulas

1. Substituting values

2. Changing the subject

Lesson 2

3. Creating graphs

4. Drawing tools

5. Changing scales

3. Equations \& graphs

4. The gradient from a graph

5. Parabolas

Lesson 3

6. Gradient and slope functions
6. From a graph to an equation

7. From a table of values to an equation
1. Editing data

2. Entering formulas

3. Creating graphs

4. Gradient \& slope functions

5. Drawing tools

6. Changing scales

1. Substituting values

2. Changing the subject

3. Equations \& graphs

4. The gradient from a graph

5. Parabolas

6. From a graph to an equation

7. From a table of values to an equation 
the instructions to understand." Options were provided on the following 7-point scale: 1, extremely easy; 2, very easy; 3, quite easy; 4 , neither easy nor difficult; 5 , quite easy; 6 , very difficult; and 7, extremely difficult.

\section{Procedure}

Prior to starting the study, students were told that they would receive three lessons designed to assist them in developing their spreadsheet skills in the area of linear modeling, and that at the conclusion of the lessons they would complete a short examination containing a mathematics and computer skills section. The acquisition phase consisted of three self-contained lessons, which were delivered on separate school days. All instructions for the study were delivered to the participants via the booklets, in which students wrote all answers to the acquisition tasks set. Each student had access to a computer. While some participants moved through certain lessons more quickly, all participants completed the activities in the required time. Students who were unsure of the processes required for each task were verbally encouraged by the teacher to go back over methods from previous lessons, but were not given any additional help. Each booklet was fully self-contained and designed for use by a novice, requiring no explanation from the teacher, with the exception of a reminder "to read everything very carefully" and a check to ensure that all participants knew how to save their work into their own personal folder. Students simply followed the instructions, performing the required tasks on a computer. Students collected the required materials at the beginning of each of the three lessons, worked individually at the computer, and handed in the booklet at the conclusion of each lesson. Reminder prompts via the relevant page were also given throughout the booklets.

During the next scheduled mathematics period following the final acquisition lesson, students completed the test phase. At the conclusion of the test phase students were required to rate how easy or difficult to understand they found the instructions. All answers were recorded on paper.

\section{RESULTS}

Because of the extended nature of the experiment (four lessons), absenteeism led to four students not completing all tasks. These students were excluded from the study, leaving 9 students in the sequential group and 11 in the concurrent group. Because of the small sample size and the initial assessment that all students were of similar mathematical ability, the 4 matched partners of the excluded students were not excluded. Mean scores for each group, on both mathematical and spreadsheet tasks in the test phase, as well as the self-rating cognitive load measures, were recorded (see Table 2). These scores were analyzed using $2 \times 2$ analysis of variance (ANOVA) with instructional group (sequential or concurrent) and spreadsheet knowledge (high or low) as the independent variables. The dependent variables were scores on both mathematical and spreadsheet knowledge tests, and subjective rating scores.

\section{Performance Scores}

Mathematics test scores. For the mathematics test scores there were no main effects for instructional group, $F(1,15)=1.91, M S E=5.06, p=0.19$; nor spreadsheet ability, $F(1,15)=0.01, M S E=$ $0.02, p=0.93$. However, there was an interaction between instructional group and spreadsheet ability, $F(1,15)=4.72, M S E=12.97, p=0.04, \eta^{2}=$ 0.25 . Simple effects tests indicated that the less experienced spreadsheet group scored higher on the math test if they received sequential instruction compared with a concurrent format: $t(6)=2.57, p=0.04$. However, for the more experienced spreadsheet group there was no significant difference found.

Spreadsheet test scores. For the spreadsheet test scores there were no significant main effects for instructional group, $F(1,15)=1.46, M S E=3.29, p$ $=0.24$; nor spreadsheet ability, $F(1,15)=0.81$ $M S E=1.81, p=0.38$. There was also no interaction between instructional group and spreadsheet ability, $F(1,15)=2.47, M S E=5.56, p=0.14$.

Cognitive load scores. For the posttest cognitive 
load measure there were no main effects for instructional group, $F(1,15)=0.08, M S E=0.11, p$ $=0.78$; nor spreadsheet ability, $F(1,15)=1.912$, $M S E=2.65, p=0.19$. However, there was a significant interaction between instructional group and spreadsheet ability, $F(1,14)=6.60, M S E=$ 9.14, $p=0.03, \eta^{2}=0.29$. Simple effects tests indicated that for the more experienced spreadsheet group cognitive load was rated significantly lower in the concurrent format, $t(9)=2.45, p=$ 0.04 ; whereas for the less experienced spreadsheet group no significant difference was found, $t(6)=1.32, p=0.24$.

\section{DISCUSSION}

The main hypothesis tested in this study was supported: Students with a low-level knowledge of spreadsheets learned mathematics more effectively if the relevant spreadsheet skills were learned prior to attempting the mathematical tasks. Both measures of performance on the mathematical task and the subjective measures of cognitive load supported this interpretation. The second hypothesis, that students experienced in spreadsheets would benefit from a concurrent treatment, was not supported. Nevertheless, the results suggest that the more experienced group may possibly have been advantaged by such a treatment. Although not significant, the means for both scores on the mathematics and spreadsheet tasks are higher (see Table 2) in the concurrent group than in the sequential group. In addition, on the cognitive load measure, the simple effects test indicated that the more experienced group rated the instructional design significantly easier following the concurrent format than the sequential approach. Both sets of results suggest that experienced spreadsheet users may benefit from a concurrent approach. In terms of performance on spreadsheet tasks, no significant differences were found, although it is notable that the means for each group followed the same patterns as the means for mathematical performance.

The main findings of this study are consistent with the expertise reversal effect (see Kalyuga et al., 2003) and other research reported in this issue (see Schnotz \& Rasch; Wallen, Plass, \& Brünken) with the best instructional strategy being dependent on the levels of learner expertise. These results can be explained in CLT terms. For novices, if information is presented on both spreadsheet applications and mathematics concurrently, working memory load is excessive, and learning is inhibited compared to sequential presentation. In contrast, for learners with more expertise, presenting information on spreadsheet use in isolation has little function because they are already largely familiar with such material, and what they really need to learn is the relationship between spreadsheet knowledge and mathematics. That is best depicted in temporal contiguity in order to permit learners

Table $2 \square$ Group means and standard deviations (in brackets) of test scores and cognitive load measures

\begin{tabular}{lcccccc}
\hline & $\begin{array}{c}\text { High SA } \\
(n=5)\end{array}$ & $\begin{array}{c}\text { Sequential Group } \\
\text { Low SA } \\
(n=4)\end{array}$ & Combined & $\begin{array}{c}\text { High SA } \\
(n=7)\end{array}$ & $\begin{array}{c}\text { Concurrent Group } \\
\text { Low SA } \\
(n=4)\end{array}$ & Combined \\
\hline Test Phase Scores & & & & & & \\
Mathematics & 8.6 & 10.3 & 9.3 & 9.3 & 7.5 & 8.6 \\
& $(2.3)$ & $(1.0)$ & $(1.9)$ & $(1.1)$ & $(1.9)$ & $(1.6)$ \\
Spreadsheet & 6.8 & 8.5 & 7.6 & 8.7 & $8.3)$ & 8.6 \\
& $(1.5)$ & $(2.4)$ & $(2.0)$ & $(1.0)$ & $(1.3)$ & $(1.0)$ \\
Cognitive load measure & 2.4 & 1.8 & 2.1 & 1.0 & 3.0 & 1.7 \\
& $(1.5)$ & $(0.5)$ & $(1.2)$ & $(0.0)$ & $(1.8)$ & $(1.6)$ \\
& & & & & & \\
\hline
\end{tabular}


to more easily make important connections (see Mayer \& Anderson, 1991, 1992). Of course, an advantage in making connections is only available providing all of the information can be processed simultaneously in working memory.

The findings concerning the least experienced spreadsheet group are also consistent with the work of Kester et al. (2001, 2004, in press), who found it advantageous to present additional information sequentially rather than simultaneously. Furthermore, if there really is an advantage for more experienced learners to be presented information concurrently as the results possibly suggest, this result may also support the van Merriënboer et al. (2003) argument that low complexity material is more suitable for a concurrent approach. As expertise increases, material that is high in complexity because of high element interactivity, can be expected to reduce in effective complexity as interacting elements are subsumed into schemas.

The possibility that experienced spreadsheet learners may benefit from a concurrent approach should be a target for future research. The sample size in this study was too small to produce compelling evidence. Such research might include actual measures of spreadsheet ability rather than self-assessment. In addition to sample size, another potential weakness of this study was that subjective measures of cognitive load were collected after the final test was completed. Students were asked to rate how easy or difficult they found the instructions to understand, but having sat the final test, ratings of the instructional material may have been influenced by the test itself. Future research should introduce the subjective rating scales immediately following the instructional phases.

Based on the results of this study, the implications for e-learning are straightforward. If using technology to teach a subject area such as mathematics, the technology should be learned prior to learning the subject area. Learning both concurrently may only be effective if learners already have considerable technological knowledge. All e-learning applications require students to become familiar with the technology. Too frequently, it is assumed that such familiarity can occur simultaneously while learning a subject discipline through e-learning. Such an assumption ignores one of the basic characteristics of human cognitive architecture-our limited working memory when dealing with novel material. Students should not be required to use e-learning programs until they are thoroughly familiar with them. Only at that point, should discipline material be introduced.

Tracey Clarke, Paul Ayres [p.ayres@unsw.edu.au], and John Sweller are with the School of Education, University of New South Wales, Sydney, Australia.

Correspondence concerning this article should be addressed to Paul Ayres, University of New South Wales, School of Education, NSW2052, Sydney, Australia.

\section{REFERENCES}

Bloom, B. S. (1956) Taxonomy of educational objectives. Handbook 1: Cognitive domain. London: Longman.

Cerpa, N., Chandler, P., \& Sweller, J. (1996). Some conditions under which integrated computer-based training software can facilitate learning. Journal of Educational Computing Research, 15, 345-367.

Chandler, P., \& Sweller, J. (1996). Cognitive load while learning to use a computer program. Applied Cognitive Psychology, 10, 151-170.

Chi, M., Glaser, R., \& Rees, E. (1982). Expertise in problem solving. In R. Sternberg (Ed.), Advances in the psychology of human intelligence (pp. 7-75). Hillsdale, NJ: Erlbaum.

Cooper, G., \& Sweller, J. (1987). Effects of schema acquisition and rule automation on mathematical problem-solving transfer. Journal of Educational Psychology, 79, 347-362.

Ericsson, K. A., \& Kintsch, W. (1995). Long-term working memory. Psychological Review, 102, 211-245.

Kalyuga, S., Ayres, P., Chandler, P., \& Sweller, J. (2003). Expertise reversal effect. Educational Psychologist, 38, 23-31.

Kalyuga, S., Chandler, P., \& Sweller, J. (2001). Learner experience and efficiency of instructional guidance. Educational Psychology, 21, 5-23.

Kester, L., Kirschner, P. A., \& van Merriënboer, J. J. G. (2004). Timing of information presentation in learning statistics. Instructional Science, 32, 233-252.

Kester, L., Kirschner, P. A., \& van Merriënboer, J. J. G. (in press). The management of cognitive load during complex cognitive skill acquisition by means of computer simulated problem solving. British Journal of Educational Psychology.

Kester, L., Kirschner, P. A., van Merriënboer, J. J. G., \& Baumer, A. (2001). Just-in-time information presentation and the acquisition of complex cognitive skills. Computers in Human Behavior, 17, 373-391. 
Kotovsky, K., Hayes, J. R., \& Simon, H.A. (1985). Why are some problems hard? Evidence from Tower of Hanoi. Cognitive Psychology, 17, 248-294.

Larkin, J., McDermott, J., Simon, D., \& Simon, H. (1980). Expert and novice performance in solving physics problems. Science, 208, 1335-1342.

Mayer, R. E. (2001). Multimedia learning. New York: Cambridge University Press.

Mayer, R. E., \& Anderson, R. (1991). Animations need narrations: An experimental test of a dual-coding hypothesis. Journal of Educational Psychology, 83, 484490.

Mayer, R. E., \& Anderson, R. (1992). The instructive animation: Helping students build connections between words and pictures in multimedia learning. Journal of Educational Psychology, 84, 444-452.

Miller, G. (1956). The magical number seven, plus or minus two: Some limits on our capacity for processing information. Psychological Review, 63, 81-97.

Moreno, R., \& Valdez, A. (2005). Cognitive load and learning effects of having students organize pictures and words in multimedia environments: The role of student interactivity and feedback. [This special issue]. Educational Technology Research and Development, 53(3), 35-45.

Paas, F., Renkl, A., \& Sweller, J. (2003). Cognitive load theory and instructional design: Recent developments. Educational Psychologist, 38, 1-4.

Paas, F., Tuovinen, J., Tabbers, H., \& van Gerven, P. (2003). Cognitive load measurement as a means to advance cognitive load theory. Educational Psychologist, 38, 63-71.

Paas, F., \& van Merriënboer, J. J. G. (1993). The efficiency of instructional conditions: An approach to combine mental effort and performance measures. Human Factors, 35, 737-743.

Paas, F., \& van Merriënboer, J. J. G. (1994). Variability of worked examples and transfer of geometric problem solving skills: A cognitive load approach. Journal of Educational Psychology, 86, 122-133.

Pollock, E., Chandler, P., \& Sweller, J. (2002). Assimilating complex information. Learning and Instruction,
12, 61-86.

Renkl, A., \& Atkinson, R. (2003). Structuring the transition from example study to problem solving in cognitive skill acquisition: A cognitive load perspective. Educational Psychologist, 38, 15-22.

Schnotz, W., \& Rasch, T. (2005). Enabling, facilitating, and inhibiting effects of animations in multimedia learning: Why reduction of cognitive load can have negative results on learning. [This special issue]. Educational Technology Research and Development, 53(3), 47-58.

Sweller, J. (1988). Cognitive load during problem solving: Effects on learning. Cognitive Science, 12, 257285.

Sweller, J. (1999). Instructional design in technical areas. Melbourne, Australia: ACER Press.

Sweller, J. (2003). Evolution of human cognitive architecture. In B. Ross (Ed.), The psychology oflearning and motivation. San Diego, CA: Academic Press.

Sweller, J., \& Cooper, G. A. (1985). The use of worked examples as a substitute for problem solving in learning algebra. Cognition and Instruction, 2, 59-89.

Sweller, J., van Merriënboer, J. J. G., \& Paas, F. (1998). Cognitive architecture and instructional design. Educational Psychology Review, 10, 251-296.

Tarmizi, R., \& Sweller, J. (1988). Guidance during mathematical problem solving, Journal of Educational Psychology, 80, 424-436.

van Merriënboer, J. J. G., \& Ayres, P. (2005). Research on cognitive load theory and its design implications for e-learning. [This special issue]. Educational Technology Research and Development, 53(3), 5-13.

van Merriënboer, J. J. G., Kirschner, P., \& Kester, L. (2003). Taking the load off a learner's mind: Instructional design for complex learning. Educational Psychologist, 38, 5-13.

Wallen, E., Plass, J. L., \& Brünken, R. (2005). The function of annotations in the comprehension of scientific texts: Cognitive load effects and the impact of verbal ability. [This special issue]. Educational Technology Research and Development, 53(3), 59-71. 
Copyright of Educational Technology Research \& Development is the property of Association for Educational Communications \& Technology. The copyright in an individual article may be maintained by the author in certain cases. Content may not be copied or emailed to multiple sites or posted to a listserv without the copyright holder's express written permission. However, users may print, download, or email articles for individual use. 